\title{
COALGEBRAS IN THE DWYER-KAN LOCALIZATION OF A MODEL CATEGORY
}

\author{
MAXIMILIEN PÉROUX
}

\begin{abstract}
We show that weak monoidal Quillen equivalences induce equivalences of symmetric monoidal $\infty$-categories with respect to the Dwyer-Kan localization of the symmetric monoidal model categories. The result will induce a Dold-Kan correspondence of coalgebras in $\infty$-categories. Moreover it shows that Shipley's zig-zag of Quillen equivalences provides an explicit symmetric monoidal equivalence of $\infty$-categories for the stable Dold-Kan correspondence. We study homotopy coherent coalgebras associated to a monoidal model category and we show examples when these coalgebras cannot be rigidified. That is, their $\infty$-categories are not equivalent to the Dwyer-Kan localizations of strict coalgebras in the usual monoidal model categories of spectra and of connective discrete $R$-modules.
\end{abstract}

\section{INTRODUCTION}

Let $\mathrm{M}$ be a model category and $\mathrm{W}$ its morphism class of weak equivalences. Recall that the homotopy category $\mathrm{Ho}(\mathrm{M})$, associated to $\mathrm{M}$, is an ordinary category obtained by inverting all weak equivalences, and can also be denoted $\mathrm{M}\left[\mathrm{W}^{-1}\right]$, see Hov99, 1.2.1, 1.2.10]. However, the higher homotopy information is lost in $\mathrm{Ho}(\mathrm{M})$. Dwyer and Kan, in [DK80, suggested instead a simplicial category $\mathrm{L}^{H}(\mathrm{M}, \mathrm{W})$ sometimes called the hammock localization of $\mathrm{M}$, that retains the higher information. The idea has been translated into $\infty$ categories by Lurie in Lur17, 1.3.4.1, 1.3.4.15]. Following [Hin16, we shall prefer the term of Dwyer-Kan localization instead of underlying $\infty$-category of a model category (see motivation by Remark 2.3 below). If the model category is endowed with a symmetric monoidal structure compatible with the model structure, then the Dwyer-Kan localization is symmetric monoidal with respect to the derived tensor product.

The main result of this paper is Theorem 2.13 which shows that weak monoidal Quillen equivalences (as in SS03) lift to equivalences of symmetric monoidal $\infty$-categories with respect to the Dwyer-Kan localizations.

Any $\mathbb{A}_{\infty}$-ring spectrum is homotopic to a strictly unital and associative ring spectrum, in some monoidal model category representing spectra, say symmetric spectra. Similarly, any $\mathbb{E}_{\infty}$-ring spectrum is homotopic to a strictly unital, associative and commutative ring spectrum. See [HSS00, [MMSS01, [MM02] and EKMM97. Associative and commutative algebras in the Dwyer-Kan localization of a symmetric monoidal model category $M$ are precisely the $\mathbb{A}_{\infty}$-algebras and $\mathbb{E}_{\infty}$-algebras of $\mathrm{M}$, see [Lur17, 4.1.8.4, 4.5.4.7].

In this paper, we answer the following question: can $\mathbb{A}_{\infty}$-coalgebras and $\mathbb{E}_{\infty}$-coalgebras in spectra be homotopic to strictly counital, coassociative and cocommutative coalgebras over the sphere spectrum? In other words, can we rigidify the comultiplication in spectra? We show in Corollary 5.5 that $\mathbb{A}_{\infty}$-coalgebras cannot be rigidified to strictly coassociative and counital coalgebras in spectra, following a previous result in PS19.

A consequence of Theorem 2.13 is that it provides a Dold-Kan correspondence for $\mathbb{A}_{\infty}$ and $\mathbb{E}_{\infty}$-coalgebras in the Dwyer-Kan localization of simplicial modules and non-negatively graded chain complexes. At the level of model categories, it was shown in Sor19] that the Dold-Kan correspondence does not lift to a Quillen equivalence between simplicial coalgebras and chain coalgebras. Therefore, we show in Corollary 4.3 that $\mathbb{A}_{\infty}$-coalgebras in connective modules over an Eilenberg-Mac Lane spectrum of a commutative ring do not correspond to their strict analogue in either simplicial modules or non-negatively graded chain complexes. Moreover, we show in Corollary 4.4 that the zig-zag of Quillen equivalences from [Shi07. provides Lurie's equivalence in the stable Dold-Kan correspondence [Lur17, 7.1.2.13].

In Pér20c, we are interested in comodules in the Dwyer-Kan localization of non-negatively graded chain complexes over a finite product of fields and show that homotopy coherent comodules can be rigidified to strict comodules.

2010 Mathematics Subject Classification. 16T15, 18D10, 18N40, 18N70, 55P42, 55P43.

Key words and phrases. homotopy, spectrum, coalgebra, $\infty$-category, rigidification, Dold-Kan Correspondence. 
The paper is constructed as follows. In Section 2 we recall the various definitions of Dwyer-Kan localizations in the literature in anticipation of our main result Theorem 2.13. In Section 3, we provide a comparison maps between $\mathbb{A}_{\infty}$ and $\mathbb{E}_{\infty}$ coalgebras and their strict analogues. We also provide a simple example where these maps are equivalences in Proposition 3.4. In Section 4, we apply Theorem 2.13 to the weak monoidal Quillen equivalences of [SS03] and [Shi07] and show the failure of rigidification for connective discrete modules in Corollary 4.3. In Section 15, we provide a model structure for strict coalgebras in symmetric spectra following [HKRS17] and show in Theorem 5.4 that its Dwyer-Kan localization is not equivalent to the $\infty$-category of $\mathbb{A}_{\infty}$-coalgebras in spectra.

Acknowledgment. The results here are part of my $\mathrm{PhD}$ thesis Pér20b, and as such, I would like to express my gratitude to my advisor Brooke Shipley for her help and guidance throughout the years. I would also like to thank Ben Antieau and Tasos Moulinos for helpful discussions in the early process of writing this paper.

\section{The Dwyer-Kan Localization of a Model Category}

We show here our main result which is Theorem 2.13. We recall the definition of Dwyer-Kan localization following [Hin16], [NS18] and Lur17.

2.1. The General Definition. We begin with the general definition of the Dwyer-Kan localization of an $\infty$-category.

Definition 2.1 ([Lur17, 1.3.4.1]). Let $C$ be an $\infty$-category and fix a collection $\mathscr{W} \subseteq \operatorname{Hom}_{\mathrm{s} S e t}\left(\Delta^{1}, C\right)$ of morphisms in $C$. The Dwyer-Kan localization of $C$ with respect to the collection $\mathscr{W}$ is an $\infty$-category, denoted $C\left[\mathscr{W}^{-1}\right]$, together with a functor $f: C \rightarrow C\left[\mathfrak{W}^{-1}\right]$ that respects the following universal property.

$(\mathrm{U})$ For any other $\infty$-category $\mathscr{D}$, the functor $f$ induces an equivalence of $\infty$-categories:

$$
\mathscr{F} \text { un }\left(C\left[\mathfrak{W}^{-1}\right], \mathscr{D}\right) \stackrel{\simeq}{\longrightarrow} \operatorname{un}^{\mathscr{W}}(C, \mathscr{D}),
$$

where $\mathcal{F}_{\mathfrak{u}} \mathfrak{W}(C, \mathscr{D})$ is the full subcategory of functors $C \rightarrow \mathscr{D}$ that sends morphisms in $\mathscr{W}$ to equivalences in $\mathscr{D}$.

The Dywer-Kan localization $C\left[\mathfrak{W}^{-1}\right]$ always exists, for any choice of $C$ and $\mathfrak{W}$, see [Lur17, 1.3.4.2], and is unique up to a contractible choice. We shall be interested in the case when $C$ equals $\mathscr{N}(\mathrm{M})$, the nerve of a model category $\mathrm{M}$.

Definition 2.2 (Lur17, 1.3.4.15]). Let $M$ be a model category and $W$ its class of weak equivalences. We call $\mathscr{N}(\mathrm{M})\left[\mathrm{W}^{-1}\right]$ the Dwyer-Kan localization of $\mathrm{M}$ with respect to $\mathrm{W}$ as in Definition 2.1] where we abuse notation and let $\mathrm{W}$ denote the induced class of morphisms in the nerve $\mathscr{N}(\mathrm{M})$.

Notice that the homotopy category of $\mathscr{N}(\mathrm{M})\left[\mathrm{W}^{-1}\right]$ is precisely the homotopy category $\mathrm{Ho}(\mathrm{M})$ associated to $\mathrm{M}$ as in Hov99, 1.2.1, 1.2.10].

Remark 2.3. We do not define the hammock localization $L^{H}(M, W)$, but invite the interested reader to look for the explicit definition in [DK80, 2.1]. Since simplicial categories represent $\infty$-categories, a hammock localized simplicial category $\mathrm{L}^{H}(\mathrm{M}, \mathrm{W})$ is a model for the Dwyer-Kan localization $\mathscr{N}(\mathrm{M})\left[\mathrm{W}^{-1}\right]$. More precisely, by [Lur09, 2.2.5.1], there is a Quillen equivalence between the category of simplicial sets sSet endowed with the Joyal model structure and the category of simplicial categories sCat endowed with the Bergner model structure (see [Ber07]):

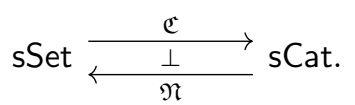

The functor $\mathfrak{N}: \mathrm{s} C a t \rightarrow \mathrm{sSet}$ is the homotopy coherent nerve, or the simplicial nerve, as in [Lur09, 1.1.5.5]. After a fibrant replacement, the functor $\mathfrak{N}$ sends $\mathrm{L}^{H}(\mathrm{M}, \mathrm{W})$ to the equivalence class of $\mathscr{N}(\mathrm{M})\left[\mathrm{W}^{-1}\right]$, as seen in [Hin16, 1.3.1].

Remark 2.4. As noted in [Lur17, 1.3.4.16], [Hin16, 1.3.4], and [DK80, 8.4], if the model category M admits functorial fibrant and cofibrant replacement, in the sense of [Hov99, 1.1.1. 1.1.3], then the following $\infty$ categories are equivalent:

$$
\mathscr{N}\left(\mathrm{M}_{c}\right)\left[\mathrm{W}^{-1}\right] \simeq \mathscr{N}(\mathrm{M})\left[\mathrm{W}^{-1}\right] \simeq \mathscr{N}\left(\mathrm{M}_{f}\right)\left[\mathrm{W}^{-1}\right],
$$


where $\mathrm{M}_{c} \subseteq \mathrm{M}$ is the full subcategory of cofibrant objects, and $\mathrm{M}_{f} \subseteq \mathrm{M}$ is the full subcategory of fibrant objects.

2.2. Symmetric Monoidal Dwyer-Kan Localization. We now construct the symmetric monoidal structure on the Dwyer-Kan localization of a symmetric monoidal model category M. This is a recollection of Appendix A in [NS18] and Section 4.1.7 on monoidal model categories in [Lur17.

Definition 2.5 ([Lur17, 4.1.7.4], [NS18, A.4, A.5]). Let $C^{\otimes}$ be a symmetric monoidal $\infty$-category. Let W $\subseteq \operatorname{Hom}_{\mathrm{sSet}}\left(\Delta^{1}, C\right)$ be a class of edges in $C$ that is stable under homotopy, composition and contains all equivalences. Suppose further that $\otimes: C \times C \rightarrow C$ preserves the class $\mathscr{W}$ separately in each variable. The symmetric monoidal Dywer-Kan localization of $\mathrm{C}^{\otimes}$ with respect to $\mathfrak{W}$ is a symmetric monoidal $\infty$-category, denoted $C\left[\mathfrak{W}^{-1}\right]^{\otimes}$, together with a symmetric monoidal functor $i: C^{\otimes} \rightarrow C\left[\mathscr{W}^{-1}\right]^{\otimes}$ which is characterized by the following universal property.

(U) For any other symmetric monoidal $\infty$-category $\mathscr{D}^{\otimes}$, the functor $i$ induces an equivalence of $\infty$ categories:

$$
\mathcal{F}_{\otimes}\left(C\left[\mathfrak{W}^{-1}\right]^{\otimes}, \mathscr{D}^{\otimes}\right) \stackrel{\simeq}{\longrightarrow} \mathcal{F} u n_{\otimes}^{W}\left(C^{\otimes}, D^{\otimes}\right),
$$

where $\mathscr{F} u n_{\otimes}^{\mathscr{W}}\left(C^{\otimes}, \mathscr{D}^{\otimes}\right)$ is the full subcategory of symmetric monoidal functors $C^{\otimes} \rightarrow \mathscr{D}^{\otimes}$ that sends W to equivalences.

As noticed in [NS18, A.5], the underlying $\infty$-category of the symmetric monoidal category $C\left[\mathfrak{W}^{-1}\right]^{\otimes}$ is precisely the Dwyer-Kan localization of $C$ with respect to $\mathscr{W}$ in the sense of Definition 2.1, i.e.:

$$
\left(e\left[\mathfrak{W}^{-1}\right]^{\otimes}\right)_{\langle 1\rangle} \simeq \mathcal{C}\left[\mathfrak{W}^{-1}\right] .
$$

Remark 2.6. Let $C^{\otimes}$ and $\mathfrak{W}$ be as in Definition 2.5 Given the symmetric monoidal structure $C^{\otimes} \rightarrow$ $\mathscr{N}\left(\mathrm{Fin}_{*}\right)$, products of $n$ edges in $\mathfrak{W}$ in $C$ correspond precisely, under the equivalence:

$$
e^{\times n} \simeq e_{\langle n\rangle}^{\otimes},
$$

to morphisms lying over id $\langle n\rangle$ in $\mathscr{N}\left(\mathrm{Fin}_{*}\right)$. This defines a class of edges $\mathfrak{W}^{\otimes} \subseteq \operatorname{Hom}_{\mathrm{sSet}}\left(\Delta^{1}, C^{\otimes}\right)$. Then the Dwyer-Kan localization of $C^{\otimes}$ with respect to $\mathfrak{W}^{\otimes}$, in the sense of Definition 2.1 denoted $C^{\otimes}\left[\left(\mathfrak{W}^{\otimes}\right)^{-1}\right]$, is equivalent to $C\left[\mathfrak{W}^{-1}\right]^{\otimes}$ defined above.

We would like to study the case where the underlying $\infty$-category of $C^{\otimes}$ is the Dwyer-Kan localization $\mathscr{N}(\mathrm{M})\left[\mathrm{W}^{-1}\right]$ of a model category $\mathrm{M}$. We first recall the induced symmetric monoidal structure on the nerve of a symmetric monoidal category.

Definition 2.7 (Lur17, 2.0.0.1]). Let $(\mathrm{C}, \otimes, \mathbb{I})$ be a symmetric monoidal category. Define a new category $\mathrm{C}^{\otimes}$, called the operator category of $\mathrm{C}$, as follows.

- Objects are sequences $\left(C_{1}, \ldots, C_{n}\right)$ where each $C_{i}$ is an object in $\mathrm{C}$, for all $1 \leq i \leq n$, for some $n \geq 1$. We allow the case $n=0$ and thus the empty set $\emptyset$ as a sequence.

- A morphism $\left(C_{1}, \ldots, C_{n}\right) \rightarrow\left(C_{1}^{\prime}, \ldots, C_{m}^{\prime}\right)$ in $\mathbf{C}^{\otimes}$ is a pair $\left(\alpha,\left\{f_{j}\right\}\right)$, where $\alpha$ is a map of finite sets $\alpha:\langle n\rangle \rightarrow\langle m\rangle$ and $\left\{f_{j}\right\}$ is a collection of $m$-morphisms in C:

$$
f_{j}: \bigotimes_{i \in \alpha^{-1}(j)} C_{i} \longrightarrow C_{j}^{\prime},
$$

for all $1 \leq j \leq m$. If $\alpha^{-1}(j)=\emptyset$, then $f_{j}$ is a morphism $\mathbb{I} \rightarrow C_{j}^{\prime}$.

- The composition of morphisms in $\mathrm{C}^{\otimes}$ is defined using the compositions in $\mathrm{Fin}_{*}$ and $\mathrm{C}$ together with the associativity of the symmetric monoidal structure of $\mathrm{C}$.

- The identity morphism on an object $\left(C_{1}, \ldots, C_{n}\right)$ is given by the identities in $\operatorname{Fin}_{*}$ and $\mathrm{C}:\left(\mathrm{id}_{\langle n\rangle},\left\{\mathrm{id}_{C_{j}}\right\}\right)$.

We obtain a functor:

$$
\mathrm{C}^{\otimes} \longrightarrow \mathrm{Fin}_{*}
$$

that sends $\left(C_{1}, \ldots C_{n}\right)$ to $\langle n\rangle$. The induced functor $\mathscr{N}\left(\mathrm{C}^{\otimes}\right) \rightarrow \mathscr{N}\left(\mathrm{Fin}_{*}\right)$ in $\infty$-categories is coCartesian and defines a symmetric monoidal structure. 
Proposition 2.8 ([Lur17, 2.1.2.21]). Given $(\mathrm{C}, \otimes, \mathbb{I})$ a symmetric monoidal category, let $\mathrm{C}^{\otimes}$ be the operator category of $\mathrm{C}$. Then the nerve $\mathscr{N}\left(\mathrm{C}^{\otimes}\right)$ is a symmetric monoidal $\infty$-category whose underlying $\infty$-category is $\mathscr{N}(\mathrm{C})$.

Remark 2.9. Let us provide the coCartesian lifts for $\mathscr{N}\left(\mathrm{C}^{\otimes}\right) \rightarrow \mathscr{N}\left(\mathrm{Fin}_{*}\right)$. Given $\left(C_{1}, \ldots, C_{n}\right)$ in $\mathrm{C}^{\otimes}$, and $\alpha:\langle n\rangle \rightarrow\langle m\rangle$ a map in $\mathrm{Fin}_{*}$, the associated coCartesian lift is induced by defining $C_{j}^{\prime}$ as follows:

$$
C_{j}^{\prime}:=\bigotimes_{i \in \alpha^{-1}(j)} C_{i},
$$

for each $1 \leq j \leq m$. Define $C_{j}^{\prime}=\mathbb{I}$ whenever $\alpha^{-1}(j)=\emptyset$. This determines a morphism $\left(C_{1}, \ldots, C_{n}\right) \rightarrow$ $\left(C_{1}^{\prime}, \ldots, C_{m}^{\prime}\right)$ in the operator category $\mathrm{C}^{\otimes}$ as desired.

If the symmetric monoidal category $(\mathrm{C}, \otimes, \mathbb{I})$ happens to be endowed with a model structure, the bifunctor $\otimes: C \times C \rightarrow C$ need not preserve weak equivalences in either variable. We need to restrict to (symmetric) monoidal model categories as in [Hov99, 4.2.6]. In any monoidal model category $(\mathrm{M}, \otimes, \mathbb{I})$, the tensor $\otimes$ : $\mathrm{M} \times \mathrm{M} \rightarrow \mathrm{M}$ preserves weak equivalences in each variable, if we restrict to cofibrant objects $\mathrm{M}_{c} \subseteq \mathrm{M}$. Moreover. the tensor product of cofibrant objects is again cofibrant. In model categories, this allows us to define a derived tensor product for the homotopy category $\mathrm{Ho}(\mathrm{M})=\mathrm{M}\left[\mathrm{W}^{-1}\right.$ ], see [Hov99, 4.3.2]. In higher category, the transition between the tensor product and the derived tensor product is exactly through the Dwyer-Kan localization of a symmetric monoidal $\infty$-category as in Definition 2.5. If we suppose in addition that $\mathbb{I}$ is cofibrant, then, as in Definition 2.7, we can define $\mathrm{M}_{c}^{\otimes} \subseteq \mathrm{M}^{\otimes}$ from the full subcategory of cofibrant objects $\mathrm{M}_{c} \subseteq \mathrm{M}$, since $\left(\mathrm{M}_{c}, \otimes, \mathbb{I}\right)$ is symmetric monoidal.

Proposition 2.10 ([Lur17, 4.1.7.6], [NS18, A.7]). Let $(\mathrm{M}, \otimes, \mathbb{I})$ be a symmetric monoidal model category. Suppose that $\mathbb{I}$ is cofibrant. Then the Dwyer-Kan localization $\mathscr{N}\left(\mathrm{M}_{c}\right)\left[\mathrm{W}^{-1}\right]$ of $\mathrm{M}$ can be given the structure of symmetric monoidal $\infty$-category via the symmetric monoidal Dwyer-Kan localization of $\mathscr{N}\left(\mathrm{M}_{c}^{\otimes}\right)$ in the sense of Definition 2.5,

$$
\mathscr{N}\left(\mathrm{M}_{c}^{\otimes}\right) \longrightarrow \mathscr{N}\left(\mathrm{M}_{c}\right)\left[\mathrm{W}^{-1}\right]^{\otimes},
$$

where $\mathrm{W}$ is the class of weak equivalences restricted to cofibrant objects in $\mathrm{M}$.

Remark 2.11. We warn the reader of the following technicality. The inclusion of cofibrant objects $M_{c} \subseteq M$ induces a lax symmetric monoidal functor $\mathscr{N}\left(\mathrm{M}_{c}^{\otimes}\right) \rightarrow \mathscr{N}\left(\mathrm{M}^{\otimes}\right)$. From Remark 2.4. Proposition 2.10 implies that we can also construct a symmetric monoidal $\infty$-category $\mathscr{N}(\mathrm{M})\left[\mathrm{W}^{-1}\right]^{\otimes}$ whose fiber over $\langle 1\rangle$ is precisely $\mathscr{N}(\mathrm{M})\left[\mathrm{W}^{-1}\right]$. However, cofibrant replacement induces a functor $\mathscr{N}\left(\mathrm{M}^{\otimes}\right) \rightarrow \mathscr{N}(\mathrm{M})\left[\mathrm{W}^{-1}\right]^{\otimes}$ that is only lax symmetric monoidal and does not share the same properties of universality as in Definition 2.5. We invite the interested reader to look at [NS18, A.7] for more details.

2.3. Weak Monoidal Quillen Equivalence. Given model categories $C$ and D, denote $W_{C}$ and $W_{D}$ their respective class of weak equivalences. Suppose we are given a Quillen adjunction:

$$
L: \mathrm{C} \underset{\perp}{\longleftarrow} \mathrm{D}: R
$$

Then as the left adjoint functor $L$ preserves weak equivalences between cofibrant objects and the right adjoint functor $R$ preserves weak equivalences between fibrant objects, we get, by [Hin16, 1.5.1], a pair of adjoint functors in $\infty$-categories between the Dwyer-Kan localizations of C and D:

$$
\mathbb{L}: \mathscr{N}(\mathrm{C})\left[\mathrm{W}_{\mathrm{C}}^{-1}\right] \underset{\perp}{\longleftarrow} \mathscr{N}(\mathrm{D})\left[\mathrm{W}_{\mathrm{D}}^{-1}\right]: \mathbb{R},
$$

where $\mathbb{L}$ and $\mathbb{R}$ represent the derived functors of $L$ and $R$. If $C$ and $\mathrm{D}$ are symmetric monoidal model categories, we investigate when the derived functors are symmetric monoidal functors of $\infty$-categories.

Definition 2.12 ([SS03, 3.6]). Let $(\mathrm{C}, \otimes, \mathbb{I})$ and $(\mathrm{D}, \wedge, \mathbb{J})$ be symmetric monoidal model categories. A weak monoidal Quillen pair consists of a Quillen adjunction:

$$
L:(\mathrm{C}, \otimes, \mathbb{I}) \underset{\perp}{\longleftarrow}(\mathrm{D}, \wedge, \mathbb{J}): R,
$$

where $L$ is lax comonoidal such that the following two conditions hold. 
(i) For all cofibrant objects $X$ and $Y$ in C, the comonoidal map:

$$
L(X \otimes Y) \longrightarrow L(X) \wedge L(Y),
$$

is a weak equivalence in $\mathrm{D}$.

(ii) For some (hence any) cofibrant replacement $\lambda: c \mathbb{I} \stackrel{\sim}{\longrightarrow} \mathbb{I}$ in $\mathrm{C}$, the composite map:

$$
L(c \mathbb{I}) \stackrel{L(\lambda)}{\longrightarrow} L(\mathbb{I}) \longrightarrow \mathbb{J},
$$

is a weak equivalence in $\mathrm{D}$, where the unlabeled map is the natural comonoidal structure of $L$.

A weak monoidal Quillen pair is a weak monoidal Quillen equivalence if the underlying Quillen pair is a Quillen equivalence.

Theorem 2.13. Let $(\mathrm{C}, \otimes, \mathbb{I})$ and $(\mathrm{D}, \wedge, \mathbb{J})$ be symmetric monoidal model categories with cofibrant units. Let $\mathrm{W}_{\mathrm{C}}$ and $\mathrm{W}_{\mathrm{D}}$ be the classes of weak equivalence in $\mathrm{C}$ and $\mathrm{D}$ respectively. Let:

$$
L:(\mathrm{C}, \otimes, \mathbb{I}) \underset{\perp}{\longleftrightarrow}(\mathrm{D}, \wedge, \mathbb{J}): R,
$$

be a weak monoidal Quillen pair. Then the derived functor of $L: \mathrm{C} \rightarrow \mathrm{D}$ induces a symmetric monoidal functor between the Dwyer-Kan localizations:

$$
\mathbb{L}: \mathscr{N}\left(\mathrm{C}_{c}\right)\left[\mathrm{W}_{\mathrm{C}}^{-1}\right] \longrightarrow \mathscr{N}\left(\mathrm{D}_{c}\right)\left[\mathrm{W}_{\mathrm{D}}^{-1}\right],
$$

where $\mathrm{C}_{c} \subseteq \mathrm{C}$ and $\mathrm{D}_{c} \subseteq \mathrm{D}$ are the full subcategories of cofibrant objects. It is the left adjoint of the derived functor of $R: \mathrm{D} \rightarrow \mathrm{C}$. If $L$ and $R$ form a weak monoidal Quillen equivalence, then $\mathbb{L}$ is a symmetric monoidal equivalence of $\infty$-categories.

Proof. Let $\mathrm{C}_{c}^{\otimes}$ and $\mathrm{D}_{c}^{\otimes}$ be the operator categories (Definition 2.7). Denote the symmetric monoidal DwyerKan localizations (Definition 2.5) by:

$$
i_{\mathrm{C}}: \mathscr{N}\left(\mathrm{C}_{c}^{\otimes}\right) \longrightarrow \mathscr{N}\left(\mathrm{C}_{c}\right)\left[\mathrm{W}_{\mathrm{C}}^{-1}\right]^{\otimes}, \quad i_{\mathrm{D}}: \mathscr{N}\left(\mathrm{D}_{c}^{\otimes}\right) \longrightarrow \mathscr{N}\left(\mathrm{D}_{c}\right)\left[\mathrm{W}_{\mathrm{D}}^{-1}\right]^{\otimes},
$$

and denote their coCartesian fibrations by:

$$
p: \mathscr{N}\left(\mathrm{C}_{c}\right)\left[\mathrm{W}_{\mathrm{C}}^{-1}\right]^{\otimes} \longrightarrow \mathscr{N}\left(\mathrm{Fin}_{*}\right), \quad q: \mathscr{N}\left(\mathrm{D}_{c}\right)\left[\mathrm{W}_{\mathrm{D}}^{-1}\right]^{\otimes} \longrightarrow \mathscr{N}\left(\mathrm{Fin}_{*}\right) .
$$

The functor $L: \mathrm{C} \rightarrow \mathrm{D}$, as a left Quillen functor, defines $\mathscr{N}\left(\mathrm{C}_{c}\right) \rightarrow \mathscr{N}\left(\mathrm{D}_{c}\right)$, and hence a functor $L^{\otimes}$ : $\mathscr{N}\left(\mathrm{C}_{c}^{\otimes}\right) \rightarrow \mathscr{N}\left(\mathrm{D}_{c}^{\otimes}\right)$ that is compatible with the coCartesian structures:

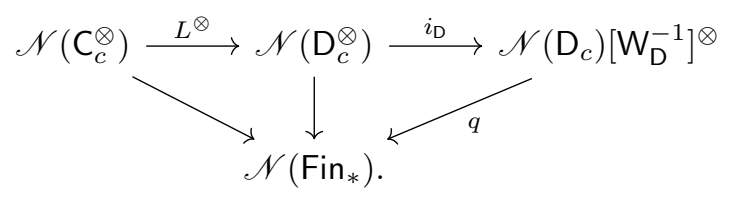

We show that the composite:

$$
\mathscr{N}\left(\mathrm{C}_{c}^{\otimes}\right) \stackrel{L^{\otimes}}{\longrightarrow} \mathscr{N}\left(\mathrm{D}_{c}^{\otimes}\right) \stackrel{i_{\mathrm{D}}}{\longrightarrow} \mathscr{N}\left(\mathrm{D}_{c}\right)\left[\mathrm{W}_{\mathrm{D}}^{-1}\right]^{\otimes}
$$

is a symmetric monoidal functor that sends $W_{C}$ to equivalences, i.e., belongs to the $\infty$-category:

$$
\mathscr{F}_{\ln }^{\mathrm{W}_{\otimes}}\left(\mathscr{N}\left(\mathrm{C}_{c}^{\otimes}\right), \mathscr{N}\left(\mathrm{D}_{c}\right)\left[\mathrm{W}_{\mathrm{D}}^{-1}\right]^{\otimes}\right)
$$

as in Definition 2.5. The latter is clear as $L$ is a left Quillen functor. We are left to show that the composite sends $p$-coCartesian lifts to $q$-coCartesian lifts. Let $\left(C_{1}, \ldots, C_{n}\right)$ be an object of $\mathbf{C}_{c}^{\otimes}$, and let $\alpha:\langle n\rangle \rightarrow\langle m\rangle$ be a morphism of finite sets. A $p$-lift of $\left(\alpha,\left(C_{1}, \ldots, C_{n}\right)\right)$ is given in Remark 2.9 by a certain sequence $\left(C_{1}^{\prime}, \ldots, C_{m}^{\prime}\right)$ in $\mathrm{C}_{c}^{\otimes}$, i.e., the induced map $\left(C_{1}, \ldots, C_{n}\right) \rightarrow\left(C_{1}^{\prime}, \ldots, C_{m}^{\prime}\right)$ is sent to $\alpha$ via the coCartesian functor $p$. Since $L$ is weak monoidal functor, from (i) of Definition 2.12, we get that:

$$
\bigwedge_{i \in \alpha^{-1}(j)} L\left(C_{i}\right) \stackrel{\sim}{\longleftarrow} L\left(\bigotimes_{i \in \alpha^{-1}(j)} C_{i}\right)=L\left(C_{j}^{\prime}\right),
$$


is a weak equivalence in $\mathrm{D}$, for all $1 \leq j \leq m$. In the case $\alpha^{-1}(j)=\emptyset$, we apply (ii) of Definition 2.12 to obtain a weak equivalence:

$$
\mathbb{J} \longleftarrow \sim(\mathbb{I})=L\left(C_{j}\right) .
$$

Applying the localization $i_{D}$ and Remark 2.6 we get that $\left(L\left(C_{1}^{\prime}\right), \ldots, L\left(C_{m}^{\prime}\right)\right)$ defines the desired $q$-coCartesian lift.

By the universal property (U) of the symmetric monoidal Dwyer-Kan localization in Definition 2.5, the composite functor $i_{\mathrm{D}} \circ L^{\otimes}$ represents a symmetric monoidal $\infty$-functor:

$$
\mathbb{L}^{\otimes}: \mathscr{N}\left(\mathrm{C}_{c}\right)\left[\mathrm{W}_{\mathrm{C}}^{-1}\right]^{\otimes} \longrightarrow \mathscr{N}\left(\mathrm{D}_{c}\right)\left[\mathrm{W}_{\mathrm{D}}^{-1}\right]^{\otimes} .
$$

Fiberwise over $\mathscr{N}\left(\mathrm{Fin}_{*}\right)$, the functor $\mathbb{L}^{\otimes}$ is precisely the product of the derived left adjoint functor $\mathbb{L}$ : $\mathscr{N}\left(\mathrm{C}_{c}\right)\left[\mathrm{W}_{\mathrm{C}}^{-1}\right] \rightarrow \mathscr{N}\left(\mathrm{D}_{c}\right)\left[\mathrm{W}_{\mathrm{D}}^{-1}\right]$. In particular, if $L$ is a Quillen equivalence, then $\mathbb{L}$ is an equivalence of $\infty$-categories, and hence $\mathbb{L}^{\otimes}$ is an equivalence of symmetric monoidal $\infty$-categories.

Remark 2.14. In [SS03, 3.12], Schwede and Shipley show that given a weak monoidal Quillen equivalence:

$$
L:(\mathrm{C}, \otimes, \mathbb{I}) \underset{\perp}{\longleftrightarrow}(\mathrm{D}, \wedge, \mathbb{J}): R,
$$

with cofibrant units, then the right adjoint $R$ induces Quillen equivalences between the category of monoids Mon(D) and Mon(C). Our Theorem 2.13 strengthen the results when we work with $\infty$-categories. In particular, given any $\infty$-operad $\mathcal{O}^{\otimes}$, we get an equivalence of $\infty$-categories:

$$
\mathcal{A} g_{\Theta}\left(\mathscr{N}\left(\mathrm{C}_{c}\right)\left[\mathrm{W}_{\mathrm{C}}^{-1}\right]\right) \simeq \mathcal{A} \mathcal{l} g_{\Theta}\left(\mathscr{N}\left(\mathrm{D}_{c}\right)\left[\mathrm{W}_{\mathrm{D}}^{-1}\right]\right)
$$

which has been challenging to prove in the case of $\mathcal{O}=\mathbb{E}_{\infty}$ in the past, see for instance [RS17] and [Man03, $1.3,1.4]$. We also obtain an equivalence on the $\infty$-categories of coalgebras (see definition in [Pér20a, 2.1]):

$$
\operatorname{Co}^{A} g_{g_{\Theta}}\left(\mathscr{N}\left(\mathrm{C}_{c}\right)\left[\mathrm{W}_{\mathrm{C}}^{-1}\right]\right) \simeq \operatorname{Co} \mathcal{A} g_{\mathcal{\Theta}}\left(\mathscr{N}\left(\mathrm{D}_{c}\right)\left[\mathrm{W}_{\mathrm{D}}^{-1}\right]\right) \text {. }
$$

Such a result on coalgebras has been shown to be untrue in some model categories, see for instance [Sor19] and Section 4.2 below.

\section{The Rigidification Problem}

In this section, we want to compare homotopy coherent coalgebras with their strict analogues. On one hand, given a nice enough symmetric monoidal model category $\mathrm{M}$, we can obtain its Dwyer-Kan localization which is a symmetric monoidal $\infty$-category. We can then define the $\infty$-category of $\mathbb{A}_{\infty}$ or $\mathbb{E}_{\infty}$-coalgebras as in Pér20a, 2.1]. On the other hand, we can consider coalgebras (also called comonoids) in the monoidal category $\mathrm{M}$ in the classical sense and then take their Dwyer-Kan localization as in Definition 2.1. We are interested to know if the $\infty$-categories are equivalent.

There are classical rigidification results that compare $\mathbb{A}_{\infty}$-algebras with their strict associative analogue, see [Lur17, 4.1.8.4]. There is also a comparison between the $\mathbb{E}_{\infty}$-case with the commutative case in [ur17, 4.5.4.7]. However, there is no reason to expect that these results dualize in general. In particular, if $\mathbb{A}_{\infty}$ algebras can be rigidified to strict associative algebras in a model category $M$, there is no reason to expect that $\mathbb{A}_{\infty}$-coalgebras correspond to strict coassociative coalgebras in $\mathrm{M}$ as we shall see in Corollary 4.3 and Theorem 5.4 below.

3.1. Rigidification Properties. Let $C$ be a (symmetric) monoidal category and denote $C^{\otimes}$ its operator category, as in Definition 2.7 Let $p: \mathrm{C}^{\otimes} \rightarrow \Delta^{\text {op }}$ be its associated Grothendieck opfibration (see [Gro20, 4.5]) that determines the monoidal structure of $\mathrm{C}$, and induces the coCartesian fibration $\mathscr{N}\left(\mathrm{C}^{\otimes}\right) \rightarrow \mathscr{N}\left(\Delta^{\mathrm{op}}\right)$. There is a correspondence between monoids in $\mathrm{C}$ and sections of $p$ that send convex morphisms to $p$ coCartesian arrows (see Gro20, 4.21]). In particular, we obtain the following equivalence of $\infty$-categories:

$$
\mathscr{N}(\operatorname{Mon}(\mathrm{C})) \longrightarrow \mathcal{A} \ell g_{\mathbb{A}_{\infty}}(\mathscr{N}(\mathrm{C}))
$$

By using opposite categories, we obtain therefore an equivalence:

$$
\mathscr{N}(\operatorname{CoA} \lg (\mathrm{C})) \longrightarrow \operatorname{Co}_{6} \mathcal{A} \lg _{\mathbb{A}_{\infty}}(\mathscr{N}(\mathrm{C})) .
$$


Let $\mathrm{M}$ be a symmetric monoidal model category with cofibrant unit. Consider $\mathrm{M}_{c} \subseteq \mathrm{M}$ the full subcategory of cofibrant objects. Apply the above identification to $C=M_{c}$ to obtain the following equivalence in $\infty$ categories:

$$
\mathscr{N}\left(\operatorname{CoAlg}\left(\mathrm{M}_{c}\right)\right) \longrightarrow \operatorname{Co} \mathcal{A} g_{\mathbb{A}_{\infty}}\left(\mathscr{N}\left(\mathrm{M}_{c}\right)\right)
$$

Let $\mathrm{W}$ be the class of weak equivalences in $\mathrm{M}$. By Proposition 2.10 there is a symmetric monoidal functor $\mathscr{N}\left(\mathrm{M}_{c}^{\otimes}\right) \rightarrow \mathscr{N}\left(\mathrm{M}_{c}\right)\left[\mathrm{W}^{-1}\right]^{\otimes}$, which thus provides a map of $\infty$-categories:

$$
\operatorname{Co} \mathcal{A} g_{\mathbb{A}_{\infty}}\left(\mathscr{N}\left(\mathrm{M}_{c}\right)\right) \longrightarrow \operatorname{Co} \mathcal{A} g_{\mathbb{A}_{\infty}}\left(\mathscr{N}\left(\mathrm{M}_{c}\right)\left[\mathrm{W}^{-1}\right]\right),
$$

and therefore we obtain a functor of $\infty$-categories:

$$
\alpha: \mathscr{N}\left(\operatorname{CoAlg}\left(\mathrm{M}_{c}\right)\right) \longrightarrow \operatorname{Co} \mathcal{A} g_{\mathbb{A}_{\infty}}\left(\mathscr{N}\left(\mathrm{M}_{c}\right)\left[\mathrm{W}^{-1}\right]\right)
$$

Denote $W_{\text {CoAlg }}$ the class of morphisms in $\operatorname{CoAlg}\left(M_{c}\right)$ that are weak equivalences as underlying morphisms in $M$. Notice that the above functor $\alpha$ sends $W_{\text {CoAlg }}$ to equivalences. By the universal property of Dwyer-Kan localizations as in Definition 2.1, we obtain the following natural functor of $\infty$-categories:

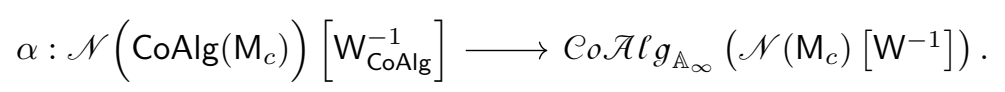

Similarly, for the cocommutative case we obtain the natural functor of $\infty$-categories:

$$
\beta: \mathscr{N}\left(\operatorname{CoCAlg}\left(\mathrm{M}_{c}\right)\right)\left[\mathrm{W}_{\text {CoCAlg }}^{-1}\right] \longrightarrow \operatorname{Co} \mathcal{A} g_{g_{\mathbb{E}_{\infty}}}\left(\mathscr{N}\left(\mathrm{M}_{c}\right)\left[\mathrm{W}^{-1}\right]\right)
$$

Definition 3.1. Let $\mathrm{M}$ be a symmetric monoidal model category with cofibrant unit. Let $\alpha$ and $\beta$ be the functors described above. If $\alpha$ is an equivalence of $\infty$-categories, we say that the model category $M$ (or its Dwyer-Kan localization) satisfies the coassociative rigidification. If $\beta$ is an equivalence of $\infty$-categories, we say that M (or its Dwyer-Kan localization) satisfies the cocommutative rigidification.

Remark 3.2. In general there is no reason to expect that if a model category $M$ respects the associative rigidification then it also respects the coassociative rigidification. We see examples below in Corollaries 4.3 and 5.5. Since coalgebras in $M$ are algebras in $M^{o p}$ this might be surprising to the reader. However, a key requirement for the associative rigidification is for $\mathrm{M}$ to be presentable, see [Lur17, 4.1.8.4, 4.5.4.7]. But if $\mathrm{M}$ is presentable, then $\mathrm{M}^{\mathrm{op}}$ is not presentable, unless $\mathrm{M}$ is a complete lattice, see [AR94, 1.64].

Remark 3.3. If we inspect the dual case of algebras [Lur17, 4.1.8.4, 4.5.4.7], we see that we should have considered the $\infty$-category $\mathscr{N}(\operatorname{CoAlg}(\mathrm{M}))\left[\mathrm{W}_{\text {CoAlg }}^{-1}\right]$ and not the $\infty$-category $\mathscr{N}\left(\operatorname{CoAlg}\left(\mathrm{M}_{c}\right)\right)\left[\mathrm{W}_{\text {CoAlg }}^{-1}\right]$. There are several issues with that.

- In general, these $\infty$-categories are not equivalent unless for instance $M$ admits a functorial lax comonoidal cofibrant replacement. This means there is a functor $Q: \mathrm{M} \rightarrow \mathrm{M}_{c}$ such that there is a natural map:

$$
Q(X \otimes Y) \rightarrow Q(X) \otimes Q(Y),
$$

for any $X$ and $Y$ in $\mathrm{M}$. The main issue is that in general the functor $\mathscr{N}\left(\mathrm{M}_{c}^{\otimes}\right) \rightarrow \mathscr{N}\left(\mathrm{M}^{\otimes}\right)$ is only lax symmetric monoidal, see Remark 2.11, Of course, if all objects in $\mathrm{M}$ are cofibrant, no such issues appear.

- There is no guarantee to have a model structure on $\operatorname{CoAlg}(M)$ whose weak equivalences are $W_{\text {CoAlg, }}$, even when using the left-induced methods from [HKRS17. Even though we do not need a model category to define $\mathscr{N}(\operatorname{CoA} \lg (\mathrm{M}))\left[\mathrm{W}_{\text {CoAlg }}^{-1}\right]$, this would help to determine if there was some compatibility with $\mathrm{M}$. For instance, if we suppose $\mathrm{M}$ is combinatorial monoidal model category and there exists a model structure on the category of coalgebra so that the forgetful-cofree adjunction:

$$
U: \operatorname{CoAlg}(\mathrm{M}) \underset{7}{\longleftarrow} \underset{\longleftarrow}{\longleftrightarrow} \mathrm{M}: \mathrm{T}^{\vee},
$$


is a Quillen adjunction, then there exists a functorial cofibrant replacement $\operatorname{CoA} \lg (\mathrm{M}) \rightarrow \operatorname{CoA} \lg \left(\mathrm{M}_{c}\right)$ that induces an equivalence of $\infty$-categories:

$$
\mathscr{N}\left(\operatorname{CoA} \lg \left(\mathrm{M}_{c}\right)\right)\left[\mathrm{W}_{\mathrm{CoAlg}}^{-1}\right] \simeq \mathscr{N}(\operatorname{CoAlg}(\mathrm{M}))\left[\mathrm{W}_{\text {CoAlg }}^{-1}\right] .
$$

- In the cases where CoAlg(M) does admit a model structure, it is in general left-induced by a model category that is not a monoidal model category. For instance, in chain complexes the left inducedmodel structure stem from the injective model structure (which is not monoidal) instead of the projective model structure, see HKRS17. See also Section 5.1 below.

All the above also applies to the cocommutative case.

3.2. The Cartesian Case. We provide here a simple case of model categories satisfying the coassociative and cocommutative rigidification in the sense of Definition 3.1 Let $(\mathrm{M}, \times, *)$ be a symmetric monoidal model category with respect to its Cartesian monoidal structure. Let $W$ be the class of weak equivalences in $M$. Suppose the terminal object $*$ is cofibrant.

Proposition 3.4. Let $(\mathrm{M}, \times, *)$ be as above. Then, $\mathrm{M}$ satisfies the coassociative and cocommutative rigidification, i.e. the following natural maps are equivalences of $\infty$-categories:

$$
\begin{aligned}
& \mathscr{N}\left(\operatorname{CoA} \lg \left(\mathrm{M}_{c}\right)\right)\left[\mathrm{W}_{\mathrm{CoAlg}}^{-1}\right] \stackrel{\simeq}{\longrightarrow} \operatorname{Co} \mathcal{A} g_{\mathbb{A}_{\infty}}\left(\mathscr{N}\left(\mathrm{M}_{c}\right)\left[\mathrm{W}^{-1}\right]\right), \\
& \mathscr{N}\left(\operatorname{CoCAlg}\left(\mathrm{M}_{c}\right)\right)\left[\mathrm{W}_{\text {CoCAlg }}^{-1}\right] \stackrel{\simeq}{\longrightarrow} \operatorname{Co} \mathcal{A} g_{\mathbb{E}_{\infty}}\left(\mathscr{N}\left(\mathrm{M}_{c}\right)\left[\mathrm{W}^{-1}\right]\right),
\end{aligned}
$$

and all four of the $\infty$-categories above are equivalent to the Dwyer-Kan localization $\mathscr{N}(\mathrm{M})\left[\mathrm{W}^{-1}\right]$.

Proof. For any Cartesian monoidal $\infty$-category $C$, we have the equivalence:

$$
\operatorname{Co} \mathcal{A} l g_{\mathbb{A}_{\infty}}(C) \simeq \operatorname{Co} \mathcal{A} g_{\mathbb{E}_{\infty}}(C) \simeq C,
$$

see [Lur17, 2.4.3.10]. For any Cartesian monoidal (ordinary) category C, we have the isomorphism of categories:

$$
\operatorname{CoAlg}(\mathrm{C}) \cong \operatorname{CoCAlg}(\mathrm{C}) \cong \mathrm{C}
$$

We obtain that the natural maps fit in the diagram:

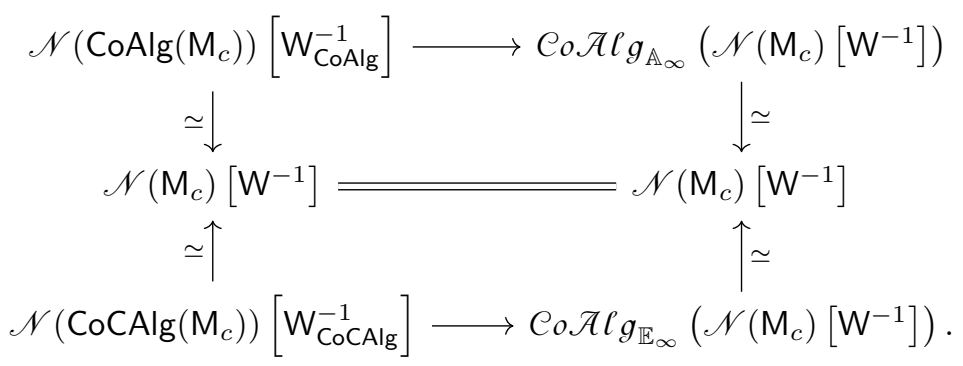

Thus we obtain our desired equivalences.

\section{Dold-Kan Correspondence For Coalgebras}

We now apply Theorem 2.13 to a weak monoidal Quillen equivalence induced by the Dold-Kan correspondence as in [SS03, all missing details can be found there. From now on, let $R$ be a commutative discrete ring. Let $\mathrm{sMod}_{R}$ denote the category of simplicial $R$-modules, and let $\mathrm{Ch}_{R}^{\geq 0}$ denote the category of non-negatively graded chain complexes. The Dold-Kan correspondence says that the normalization functor:

$$
\mathrm{N}: \mathrm{sMod}_{R} \stackrel{\cong}{\longrightarrow} \mathrm{Ch}_{\bar{R}}^{\geq 0}
$$

is an equivalence of categories. Its inverse functor is denoted $\Gamma: \mathrm{Ch}_{\bar{R}}^{\geq 0} \rightarrow \mathrm{sMod}_{R}$.

We show in Corollary 4.2 that if we derive the Dold-Kan correspondence, then we obtain a correspondence between the coalgebraic objects. Moreover, Theorem 2.13 clarifies the equivalence of the stable Dold-Kan correspondence, see Theorem 4.4. Comparing this to a result of [Sor19], we obtain that $\mathbb{A}_{\infty}$-coalgebras 
of connective modules over a discrete commutative ring cannot be rigidified in the Dold-Kan context, see Section 4.3

4.1. The Derived Dold-Kan Equivalence. We endow each category with a model structure as follows. For $\operatorname{sMod}_{R}$, the weak equivalences and fibrations are the underlying weak equivalences and fibrations in simplicial sets, i.e., they are weak homotopy equivalences and Kan fibrations. For $\mathrm{Ch}_{\bar{R}}^{\geq 0}$, we use the usual projective model structure. The weak equivalences are the quasi-isomorphisms, and the fibrations are the positive levelwise epimorphisms. Both categories can be endowed with their usual symmetric monoidal structure induced by the tensor product of $R$-modules. However, the Dold-Kan equivalence (4.1) does not preserve the monoidal structure. Nonetheless, with respect to the above choice of model structures, the categories $\operatorname{sMod}_{R}$ and $\mathrm{Ch}_{\bar{R}}^{\geq 0}$ are both symmetric monoidal model categories with cofibrant units. The isomorphism of categories from (4.1) can be regarded now as either of two Quillen equivalences, depending on the choice of left and right adjoints:

$$
\mathrm{Ch}_{\bar{R}}^{\geq 0} \underset{\mathrm{N}}{\stackrel{\Gamma}{\longleftarrow}} \operatorname{sMod}_{R}
$$

and:

$$
\operatorname{sMod}_{R} \underset{\stackrel{\mathrm{N}}{\underset{\Gamma}{\longleftarrow}}}{\stackrel{\perp}{\longrightarrow}} \mathrm{Ch}_{\bar{R}}^{\geq 0}
$$

If we choose the normalization functor $\mathrm{N}$ to be the right adjoint as in (4.2), then it can be considered as lax symmetric monoidal via the shuffle map. If we choose $\mathrm{N}$ to be the left adjoint as in (4.3), then the Alexander-Whitney formula gives a lax comonoidal structure which is not symmetric.

Nevertheless, this shows that both Quillen equivalences form a weak monoidal Quillen equivalence with cofibrant units, which is symmetric in the case where $\mathrm{N}$ is a right adjoint (4.2). We can therefore apply Theorem 2.13 to obtain the following.

Corollary 4.1 (The Derived Dold-Kan Correspondence). Let $R$ be a commutative discrete ring. Then the Dwyer-Kan localizations of $\operatorname{sMod}_{R}$ and $\mathrm{Ch}_{R}^{\geq 0}$ are equivalent as symmetric monoidal $\infty$-categories:

$$
\mathscr{N}\left(\operatorname{sMod}_{R}\right)\left[\mathrm{W}_{\Delta}^{-1}\right] \simeq \mathscr{N}\left(\mathrm{Ch}_{R}^{\geq 0}\right)\left[\mathrm{W}_{\mathrm{dg}}^{-1}\right]
$$

via the left Quillen derived functor $\Gamma: \mathrm{Ch}_{\bar{R}} \geq 0 \rightarrow \operatorname{sMod}_{R}$ from the Quillen equivalence of (4.2), where $\mathrm{W}_{\Delta}$ is the class of weak homotopy equivalences between simplicial $R$-modules, and $\mathrm{W}_{\mathrm{dg}}$ is the class of quasiisomorphisms between non-negatively graded chain complexes over $R$.

In particular, applying Remark 2.14, we get the following result.

Corollary 4.2 (Dold-Kan Correspondence For Coalgebras). For any $\infty$-operad $\mathcal{\Theta}$, the normalization functor induces an equivalence of $\infty$-categories:

$$
\operatorname{Co} \mathcal{A} g_{\Theta}\left(\mathscr{N}\left(\operatorname{sMod}_{R}\right)\left[\mathrm{W}_{\Delta}^{-1}\right]\right) \stackrel{\simeq}{\longrightarrow} \operatorname{Co} \mathcal{A} g_{g_{\Theta}}\left(\mathscr{N}\left(\mathrm{Ch}_{R}^{\geq 0}\right)\left[\mathrm{W}_{\mathrm{dg}}^{-1}\right]\right) .
$$

4.2. Rigidification Failure of Connective Coalgebras. The above result bypasses a difficulty on the level of model categories and strict coalgebras. If we choose the second adjunction (4.3) as a weak Quillen monoidal equivalence, then the normalization functor, being lax comonoidal, lifts to coalgebras:

$$
\mathrm{N}: \operatorname{CoAlg}_{R}\left(\operatorname{sMod}_{R}\right) \rightarrow \operatorname{CoAlg}_{R}\left(\mathrm{Ch}_{R}^{\geq 0}\right)
$$

but its inverse $\Gamma$, being only lax monoidal, does not lift to coalgebras. Nevertheless, a right adjoint exists on the level of $R$-coalgebras, either by presentability, or using dual methods as in section 3.3 of [SS03. We shall denote it by $\Gamma_{\text {CoAlg. }}$ Then, using left-induced methods of [HKRS17, we obtain model structures and a Quillen adjunction:

$$
\operatorname{CoAlg}_{R}\left(\operatorname{sMod}_{R}\right) \underset{\Gamma_{\text {CoAlg }}}{\stackrel{\mathrm{N}}{\rightleftarrows}} \operatorname{CoAlg}_{R}\left(\mathrm{Ch}_{R}^{\geq 0}\right)
$$

The weak equivalences are the underlying weak equivalences and every object is cofibrant, in both model categories. However, it was shown in [Sor19, 4.16] that the above Quillen pair is not a Quillen equivalence, 
at least when $R$ is a field. It was shown that for a particular choice of fibrant object $C$ in $\operatorname{CoAlg}_{R}\left(\mathrm{Ch}_{\bar{R}}^{\geq 0}\right)$, the counit $\mathrm{N}\left(\Gamma_{\text {CoAlg }}(C)\right) \longrightarrow C$ is not a weak equivalence (i.e. not a quasi-isomorphism).

Therefore, the normalization functor does not induce an equivalence of $\infty$-categories on the Dwyer-Kan localizations:

$$
\mathrm{N}: \mathscr{N}\left(\operatorname{CoAlg}\left(\mathrm{sMod}_{\mathrm{R}}\right)\right)\left[\mathrm{W}_{\Delta, \mathrm{CoAlg}}^{-1} \stackrel{Æ}{\rightarrow} \mathscr{N}\left(\mathrm{CoAlg}\left(\mathrm{Ch}_{R}^{\geq 0}\right)\right)\left[\mathrm{W}_{\mathrm{dg}, \mathrm{CoAlg}}^{-1}\right] .\right.
$$

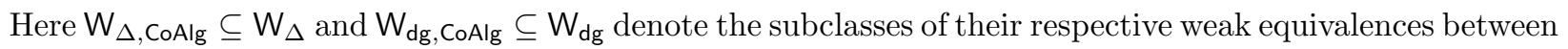
coalgebra objects. However, Corollary 4.2 shows that the normalization functor induces an equivalence between the $\mathbb{A}_{\infty}$-coalgebras:

$$
\mathrm{N}: \operatorname{Co} \mathcal{A} g_{\mathbb{A}_{\infty}}\left(\mathscr{N}\left(\operatorname{sMod}_{R}\right)\left[\mathrm{W}_{\Delta}^{-1}\right]\right) \stackrel{\simeq}{\longrightarrow} \operatorname{Co} \mathcal{A} l g_{\mathbb{A}_{\infty}}\left(\mathscr{N}\left(\mathrm{Ch}_{\bar{R}}^{>0}\right)\left[\mathrm{W}_{\mathrm{dg}}^{-1}\right]\right) .
$$

By [Lur17, 7.1.3.10], the $\infty$-categories $\mathscr{N}\left(\mathrm{sMod}_{R}\right)\left[\mathrm{W}_{\Delta}^{-1}\right]$ and $\mathscr{N}\left(\mathrm{Ch}_{\bar{R}}^{\geq 0}\right)\left[\mathrm{W}_{\mathrm{dg}}^{-1}\right]$ represent the symmetric monoidal $\infty$-category $\bmod _{\mathrm{H} R} \geq 0$ of $H R$-modules in connective spectra. From our above discussion, we obtain the following result which says that we cannot rigidify coassociative coalgebras in one of the model categories $\mathrm{Ch}_{\bar{R}}^{\geq 0}$ or $\operatorname{sMod}_{R}$.

Corollary 4.3. Let $R$ be a commutative ring. Consider $\operatorname{sMod}_{R}$ and $\mathrm{Ch}_{\bar{R}}^{\geq 0}$ with their standard monoidal model structures. Then one of these two categories, which we denote $\mathrm{M}$, does not satisfy the coassociative rigidification, i.e.:

$$
\mathscr{N}\left(\operatorname{CoA} \lg \left(\mathrm{M}_{c}\right)\right)\left[\mathrm{W}^{-1}\right] \stackrel{\varkappa}{\longrightarrow} \operatorname{Co} \mathcal{A} l g_{\mathbb{A}_{\infty}}\left(m_{o d} d_{H R}^{\geq 0}\right),
$$

where $\mathrm{W}$ is the class of maps of coalgebras which are weak equivalences in $\mathrm{M}$.

4.3. The Stable Dold-Kan Correspondence. Our approach also gives a new proof of the stable DoldKan correspondence. This well-known result was formalized with $\infty$-categories in [Lur17, 7.1.2.13] as follows. Let $R$ be a commutative discrete ring. Then the $\infty$-category of $H R$-modules in spectra $\bmod _{H R}$ is equivalent to $\infty$-category of derived $R$-modules $\mathscr{D}(R)$ as symmetric monoidal $\infty$-categories: $\bmod _{H R} \simeq \mathscr{D}(R)$. However, the equivalence was not described explicitly in [Lur17. In [Shi07, 2.10], Shipley provided an explicit zig-zag of (weak monoidal) Quillen equivalences between the standard model category $\operatorname{Mod}_{H R}$ of $H R$-modules in symmetric spectra and the projective model category of chain complexes over $R$ :

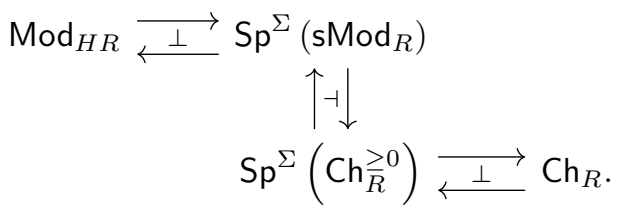

Notice that the Dwyer-Kan localizations of $\operatorname{Mod}_{H R}$ and $\mathrm{Ch}_{R}$ are precisely the $\infty$-categories $\operatorname{Mod}_{H R}$ and $\mathscr{D}(R)$ respectively. If we derive and combine the Quillen functors above, we obtain a functor of $\infty$-categories $\Theta: \bmod _{H R} \rightarrow \mathscr{D}(R)$. Recall that both $\mathrm{sMod}_{R}$ and $\mathrm{Ch}_{R}^{\geq 0}$ are left proper cellular symmetric monoidal model categories. Recall from [ABG18, B.3] that there is a compatibility with stabilization and the Dwyer-Kan localization of a left proper cellular simplicial symmetric monoidal model category. Combining the above with Corollary 4.1 and Theorem 2.13 yields the following.

Corollary 4.4 (The Stable Dold-Kan Correspondence). Let $R$ be a commutative discrete ring. Then the $\infty$-category of $H R$-modules is equivalent to the $\infty$-category of derived $R$-modules as symmetric monoidal $\infty$-categories via the functor:

$$
\Theta: \bmod _{H R} \stackrel{\simeq}{\longrightarrow} D(R) .
$$

Moreover, the functor induces an equivalence for any $\infty$-operad $\mathcal{O}$ :

$$
\operatorname{Co} \lg _{\mathcal{\Theta}}\left(\bmod _{H R}\right) \stackrel{\simeq}{\longrightarrow} \operatorname{Co} \mathcal{l} g_{\Theta}(D(R)) \text {. }
$$




\section{Coalgebras in Spectra}

Based on the main result of [PS19], we prove here, in Corollary 5.5] that the monoidal model categories of symmetric spectra (see [HSS00]), orthogonal spectra (see [MMSS01] [MM02]), Г-spaces (see [Seg74] BF78]), and $\mathscr{W}$-spaces (see And74]), do not respect the coassociative rigidification, in the sense of Definition 3.1 In other words, the strictly coassociative counital coalgebras in these monoidal categories of spectra do not have the correct homotopy type.

We work with the symmetric monoidal model category of symmetric spectra, denoted Sp ${ }^{\Sigma}$ (see [HSS00]), and claim that similar results can be obtained with the other categories mentioned above, following [PS19. Notice that we have the equivalence of $\infty$-categories:

$$
\mathscr{N}\left(\mathrm{Sp}^{\Sigma}\right)\left[\mathrm{W}^{-1}\right] \simeq \mathcal{S p}
$$

where $W$ is the class of stable equivalences of symmetric spectra, and $\delta p$ is the $\infty$-category of spectra as in Lur17, 1.4.3.1].

5.1. Model Structures for Coalgebras. Although not necessary to show the non-rigidification, as seen in Remark 3.3, we provide a model category for coalgebras and cocommutative coalgebras in symmetric spectra. We shall use the left-induced methods from [HKRS17. In [HSS00, Section 5] there is a simplicial, combinatorial model structure on $\mathrm{Sp}^{\Sigma}$ with all objects cofibrant called the (absolute) injective stable model structure, see also [Sch, Remark III.4.13].

Proposition 5.1 ([HKRS17, 5.0.1, 5.0.2]). For any $\mathbb{S}$-algebra $A$ in $\mathrm{Sp}^{\Sigma}$, there exists an injective model structure on $\operatorname{Mod}_{A}\left(\mathrm{Sp}^{\Sigma}\right)$ left-induced from the injective stable model structure on $\mathrm{Sp}^{\Sigma}$ :

$$
\operatorname{Mod}_{A}\left(\mathrm{Sp}^{\Sigma}\right) \underset{\operatorname{Hom}_{\mathrm{Sp}} \Sigma(A,-)}{\stackrel{\perp}{\overleftarrow{ }}} \mathrm{Sp}^{\Sigma}
$$

with cofibrations the monomorphisms and weak equivalences the stable equivalences. This model structure on $\operatorname{Mod}_{A}\left(\mathrm{Sp}^{\Sigma}\right)$ is simplicial and combinatorial.

Let $A$ be a commutative ring spectrum (i.e. a commutative $\mathbb{S}$-algebra). The symmetric monoidal category $\left(\operatorname{Mod}_{A}\left(\mathrm{Sp}^{\Sigma}\right), \wedge_{A}, A\right)$ is presentable and the smash product $\wedge_{A}$ preserves colimits in both variables. Thus we can apply [Por08, 2.7] and we obtain the (forgetful-cofree)-adjunction between $A$-coalgebras and $A$-modules in $\mathrm{Sp}^{\Sigma}$ :

$$
\operatorname{CoAlg}_{A}\left(\mathrm{Sp}^{\Sigma}\right) \underset{\frac{U}{\mathrm{~T}^{\vee}}}{\stackrel{\perp}{\longleftarrow}} \operatorname{Mod}_{A}\left(\mathrm{Sp}^{\Sigma}\right) .
$$

Proposition 5.2. Let $A$ be any commutative $\mathbb{S}$-algebra in symmetric spectra $\mathrm{Sp}^{\Sigma}$. There exists a combinatorial model structure on A-coalgebras $\mathrm{CoAlg}_{A}\left(\mathrm{Sp}^{\Sigma}\right)$ left-induced by the (forgetful-cofree) adjunction from the injective stable model structure on $\operatorname{Mod}_{A}\left(\mathrm{Sp}^{\Sigma}\right)$. In particular, the weak equivalences in $\operatorname{CoAlg}_{A}\left(\operatorname{Sp}^{\Sigma}\right)$ are the underlying stable equivalences, and the cofibrations are the underlying monomorphisms.

Proof. We mimic the proof of [HKRS17, Theorem 5.0.3]. We apply [HKRS17, 2.2.1]. Tensoring with a simplicial set lifts to $A$-coalgebras. Indeed, let $K$ be a simplicial set and $\left(C, \Delta_{C}, \varepsilon_{C}\right)$ be an $A$-coalgebra. Then the free $\mathbb{S}$-module $\Sigma_{+}^{\infty} K$ is endowed with a unique (cocommutative) $\mathbb{S}$-coalgebra structure $\left(\Sigma_{+}^{\infty} K, \Delta_{K}, \varepsilon_{K}\right)$, see [PS19, Lemma 2.4], where the comultiplication $\Delta_{K}$ is induced by the diagonal $K_{+} \rightarrow K_{+} \wedge K_{+}$and the counit $\varepsilon_{K}$ is induced by the non-trivial map $K_{+} \rightarrow S^{0}$. Then the tensor $K \otimes C:=\Sigma_{+}^{\infty} K \wedge_{\mathbb{S}} C$ is an $A$-coalgebra with comultiplication:

$$
\begin{aligned}
\Sigma_{+}^{\infty} K \wedge_{\mathbb{S}} C \stackrel{\Delta_{K} \wedge \Delta_{C}}{\longrightarrow} & \left(\Sigma_{+}^{\infty} K \wedge_{\mathbb{S}} \Sigma_{+}^{\infty} K\right) \wedge_{\mathbb{S}}\left(C \wedge_{A} C\right) \\
& \cong\left(\Sigma_{+}^{\infty} K \wedge_{\mathbb{S}} C\right) \wedge_{A}\left(\Sigma_{+}^{\infty} K \wedge_{\mathbb{S}} C\right),
\end{aligned}
$$

and counit:

$$
\Sigma_{+}^{\infty} K \wedge_{\mathbb{S}} C \stackrel{\varepsilon_{K} \wedge \varepsilon_{G}}{\longrightarrow} \mathbb{S} \wedge_{\mathbb{S}} A \cong A
$$

There is a good cylinder object in sSet given by the factorization:

$$
S^{0} \coprod S^{0} \longleftrightarrow \Delta[1]_{+}=I \stackrel{\sim}{\longrightarrow} S^{0} .
$$


Since $\operatorname{Mod}_{A}\left(\mathrm{Sp}^{\Sigma}\right)$ is simplicial, all objects are cofibrant, and that the smash product of an $A$-coalgebra with this factorization in sSet lifts to $\operatorname{CoAlg}_{A}\left(\mathrm{Sp}^{\Sigma}\right)$, this defines a good cylinder object in $\operatorname{CoAlg}_{A}\left(\mathrm{Sp}^{\Sigma}\right)$ for any $A$-coalgebra $C$ :

$$
C \coprod C \succ C \otimes I \stackrel{\sim}{\longrightarrow} C
$$

as $C \otimes S^{0} \cong C$, and colimits in $\operatorname{CoAlg}_{A}\left(\mathrm{Sp}^{\Sigma}\right)$ are computed in $\operatorname{Mod}_{A}\left(\mathrm{Sp}^{\Sigma}\right)$. This is a combinatorial model structure by $\left[\mathrm{BHK}^{+} 15,2.23\right]$ and [HKRS17, 3.3.4].

In the proof above, we see that the tensor $K \otimes C$ remains a cocommutative $A$-coalgebra if $C$ is cocommutative, since the coalgebra $\Sigma_{+}^{\infty} K$ is always cocommutative. Thus, we can extend the result to cocommutative A-coalgebras.

Proposition 5.3. Let $A$ be any commutative $\mathbb{S}$-algebra in symmetric spectra $\mathrm{Sp}^{\Sigma}$. There exists a combinatorial model structure on the category of cocommutative $A$-coalgebras $\operatorname{CoCAlg}_{A}\left(\mathrm{Sp}^{\Sigma}\right)$ left-induced by the (forgetful-cofree) adjunction from the injective stable model structure on $\operatorname{Mod}_{A}\left(\mathrm{Sp}^{\Sigma}\right)$. In particular, the weak equivalences in $\operatorname{CoCAlg}_{A}\left(\mathrm{Sp}^{\Sigma}\right)$ are the underlying stable equivalences, and the cofibrations are the underlying monomorphisms.

5.2. The Failure of Rigidification. We show here the failure of rigidification. Let $A$ and $B$ be commutative $\mathbb{S}$-coalgebras. A map $A \rightarrow B$ is defined to be a positive flat cofibration of commutative $\mathbb{S}$-algebras if it is a cofibration in the model category of commutative $\mathbb{S}$-algebras defined in [Shi04, 3.2] (or the positive flat stable model structure defined in [Sch, III.6.1]). Any $\mathbb{E}_{\infty}$-ring spectrum is equivalent to a positive flat cofibrant commutative $\mathbb{S}$-algebra in $\mathrm{Sp}^{\Sigma}$. As noted in [PS19, 2.4], every comonoid in $\left(\mathrm{sSet}_{*}, \wedge, S^{0}\right)$ is of the form $Y_{+}$ and the comultiplication is given by the diagonal $Y_{+} \rightarrow(Y \times Y)_{+} \cong Y_{+} \wedge Y_{+}$.

Theorem 5.4 ([PS19, 3.4, 3.6]). Let $A$ be a positive flat cofibrant commutative $\mathbb{S}$-algebra in $\mathrm{Sp}^{\Sigma}$. Then, given any counital coassociative $A$-coalgebra $C$ in $\mathrm{Sp}^{\Sigma}$, the comultiplication is cocommutative and induced by the following epimorphism of A-coalgebras:

$$
A \wedge C_{0} \longrightarrow C,
$$

where $A \wedge C_{0}$ is given an $A$-coalgebra structure via the diagonal on the pointed space $C_{0} \rightarrow C_{0} \wedge C_{0}$.

Let $A$ be any commutative $\mathbb{S}$-algebra. Let $\operatorname{CoAlg}_{A}\left(\operatorname{Sp}_{c}^{\Sigma}\right)$ denote the comonoid in the cofibrant objects of $A$-modules in $\mathrm{Sp}^{\Sigma}$ endowed with the absolute projective stable model structure (as in [Sch, IV.6.1]). There is a natural map of $\infty$-categories:

$$
\alpha: \mathscr{N}\left(\operatorname{CoAlg}_{A}\left(\mathrm{Sp}_{c}^{\Sigma}\right)\right)\left[\mathrm{W}^{-1}\right] \longrightarrow \operatorname{Co} \mathcal{A} g_{\mathbb{A}_{\infty}}\left(\bmod _{A}(\mathcal{S p})\right)
$$

where $\mathrm{W}$ is the class of stable equivalences between $A$-coalgebras.

Corollary 5.5. Let $A$ be a positive flat cofibrant commutative $\mathbb{S}$-algebra in $\mathrm{Sp}^{\Sigma}$. Then the $\infty$-category of $A$-modules $\bmod _{A}(\mathcal{S} p)$ does not satisfy the coassociative rigidification. In particular, for $A=\mathbb{S}$ we have:

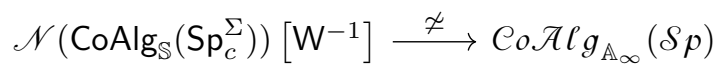

Proof. Let $(C, \Delta, \varepsilon)$ be an $A$-coalgebra in $\mathrm{Sp}^{\Sigma}$ that is cofibrant as an $A$-module in the (absolute) projective stable model structure. Suppose the functor:

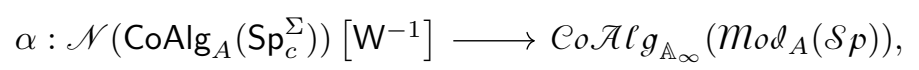

is an equivalence of $\infty$-categories. By Theorem 5.4 we see that $\alpha(C)$ is automatically an $\mathbb{E}_{\infty}$-coalgebra. But there exist $\mathbb{A}_{\infty}$-coalgebras in $\delta p$ that are not $\mathbb{E}_{\infty}$-coalgebras. Indeed, take any compact topological group that is not Abelian (say $O(2)$ ), then $A \wedge O(2)_{+}$is an $\mathbb{A}_{\infty}$-algebra in $\bmod _{A}(\mathcal{S} p$ ) that is not commutative and is a compact spectrum. By Spanier-Whitehead duality, we obtain an $\mathbb{A}_{\infty}$-coalgebra that is not $\mathbb{E}_{\infty}$ in spectra. 
Remark 5.6. Let $A$ be a positive flat cofibrant commutative $\mathbb{S}$-algebra in $\mathrm{Sp}^{\Sigma}$. Let $G$ be a compact Abelian Lie group (say a torus). Then the Spanier-Whitehead dual of the commutative ring spectrum $A \wedge G_{+}$is an $\mathbb{E}_{\infty}$-coalgebra in spectra with comultiplication that is not the diagonal. Therefore the $\infty$-category of $A$-modules $\bmod _{A}(\delta p)$ does not satisfy the cocommutative rigidification. In particular, for $A=\mathbb{S}$ we have:

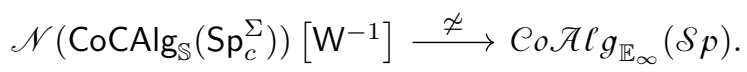

\section{REFERENCES}

[ABG18] Matthew Ando, Andrew J. Blumberg, and David Gepner, Parametrized spectra, multiplicative Thom spectra and the twisted Umkehr map, Geom. Topol. 22 (2018), no. 7, 3761-3825. MR 3890766

[And74] D. W. Anderson, Convergent functors and spectra, Localization in group theory and homotopy theory, and related topics (Sympos., Battelle Seattle Res. Center, Seattle, Wash., 1974), Lecture Notes in Math., vol. 418, Springer, Berlin, 1974, pp. 1-5. MR 0383388

[AR94] Jiří Adámek and Jiří Rosický, Locally presentable and accessible categories, London Mathematical Society Lecture Note Series, vol. 189, Cambridge University Press, Cambridge, 1994. MR 1294136

[Ber07] Julia E. Bergner, A model category structure on the category of simplicial categories, Trans. Amer. Math. Soc. 359 (2007), no. 5, 2043-2058. MR 2276611

[BF78] A. K. Bousfield and E. M. Friedlander, Homotopy theory of $\Gamma$-spaces, spectra, and bisimplicial sets, Geometric applications of homotopy theory (Proc. Conf., Evanston, Ill., 1977), II, Lecture Notes in Math., vol. 658, Springer, Berlin, 1978, pp. 80-130. MR 513569

$\left[\mathrm{BHK}^{+} 15\right]$ Marzieh Bayeh, Kathryn Hess, Varvara Karpova, Magdalena Kędziorek, Emily Riehl, and Brooke Shipley, Leftinduced model structures and diagram categories, Women in topology: collaborations in homotopy theory, Contemp. Math., vol. 641, Amer. Math. Soc., Providence, RI, 2015, pp. 49-81. MR 3380069

[DK80] W. G. Dwyer and D. M. Kan, Calculating simplicial localizations, J. Pure Appl. Algebra 18 (1980), no. 1, 17-35. MR 578563

[EKMM97] Anthony Elmendorf, Igor Kriz, Michael A. Mandell, and Peter May, Rings, modules, and algebras in stable homotopy theory, Mathematical Surveys and Monographs, vol. 47, American Mathematical Society, Providence, RI, 1997, With an appendix by M. Cole. MR 1417719

[Gro20] Moritz Groth, A short course on $\infty$-categories, Handbook of homotopy theory (Haynes Miller, ed.), CRC Press, Boca Raton, FL, 2020, pp. 549-618.

[Hin16] Vladimir Hinich, Dwyer-Kan localization revisited, Homology Homotopy Appl. 18 (2016), no. 1, 27-48. MR 3460765

[HKRS17] Kathryn Hess, Magdalena Kędziorek, Emily Riehl, and Brooke Shipley, A necessary and sufficient condition for induced model structures, J. Topol. 10 (2017), no. 2, 324-369. MR 3653314

[Hov99] Mark Hovey, Model categories, Mathematical Surveys and Monographs, vol. 63, American Mathematical Society, Providence, RI, 1999. MR 1650134

[HSS00] Mark Hovey, Brooke Shipley, and Jeff Smith, Symmetric Spectra, J. Amer. Math. Soc. 13 (2000), no. 1, $149-208$. MR 1695653

[Lur09] Jacob Lurie, Higher topos theory, Annals of Mathematics Studies, vol. 170, Princeton University Press, Princeton, NJ, 2009. MR 2522659

[Lur17] Jacob Lurie, Higher algebra, https://www.math.ias.edu/ lurie/papers/HA.pdf 2017, electronic book.

[Man03] Michael A. Mandell, Topological André-Quillen cohomology and E $\infty_{\infty}$, André-Quillen cohomology, Adv. Math. 177 (2003), no. 2, 227-279. MR 1990939

[MM02] Michael A. Mandell and Peter May, Equivariant orthogonal spectra and S-modules, Mem. Amer. Math. Soc. 159 (2002), no. $755, \mathrm{x}+108$. MR 1922205

[MMSS01] Michael A. Mandell, Peter May, Stefan Schwede, and Brooke Shipley, Model categories of diagram spectra, Proc. London Math. Soc. (3) 82 (2001), no. 2, 441-512. MR 1806878

[NS18] Thomas Nikolaus and Peter Scholze, On topological cyclic homology, Acta Math. 221 (2018), no. 2, $203-409$. MR 3904731

[Pér20a] Maximilien Péroux, The coalgebraic enrichment of algebras in higher categories, 2020, arXiv.

[Pér20b] - Highly structured coalgebras and comodules, https://homepages.math.uic.edu/ mholmb2/thesis-2.pdf 2020, Phd thesis.

[Pér20c] - Rigidificaton of connective comodules, 2020, arXiv.

[Por08] Hans-E. Porst, On categories of monoids, comonoids, and bimonoids, Quaest. Math. 31 (2008), no. 2, $127-139$. MR 2529129

[PS19] Maximilien Péroux and Brooke Shipley, Coalgebras in symmetric monoidal categories of spectra, Homology Homotopy Appl. 21 (2019), no. 1, 1-18. MR 3852287

[RS17] Birgit Richter and Brooke Shipley, An algebraic model for commutative HZ-algebras, Algebr. Geom. Topol. 17 (2017), no. 4, 2013-2038. MR 3685600

[Sch] Stefan Schwede, Symmetric spectra, http://www.math.uni-bonn.de/people/schwede/SymSpec-v3.pdf, electronic book.

[Seg74] Graeme Segal, Categories and cohomology theories, Topology 13 (1974), 293-312. MR 0353298 
[Shi04] Brooke Shipley, A convenient model category for commutative ring spectra, Homotopy theory: relations with algebraic geometry, group cohomology, and algebraic $K$-theory, Contemp. Math., vol. 346, Amer. Math. Soc., Providence, RI, 2004, pp. 473-483. MR 2066511

[Shi07] ㄴ HZl-algebra spectra are differential graded algebras, Amer. J. Math. 129 (2007), no. 2, $351-379$. MR 2306038

[Sor19] W. Hermann B. Soré, On a Quillen adjunction between the categories of differential graded and simplicial coalgebras, J. Homotopy Relat. Struct. 14 (2019), no. 1, 91-107. MR 3913972

[SS03] Stefan Schwede and Brooke Shipley, Equivalences of monoidal model categories, Algebr. Geom. Topol. 3 (2003), 287-334. MR 1997322

Department of Mathematics, University of Pennsylvania, 209 South 33rd Street, Philadelphia, PA, 19104-6395, USA

Email address: mperoux@sas.upenn.edu 